\title{
Performance estimate of some prototypes of inertial platform and strapdown marine gravimeters
}

Yuan Yuan ${ }^{1,2^{*}}$, Jinyao Gao ${ }^{1,2}$, Zhaocai Wu ${ }^{1,2}$, Zhongyan Shen ${ }^{1,2}$ and Guochao Wu ${ }^{1,2}$

\begin{abstract}
Marine gravimeter has been proved to be the primary technique to efficiently obtain middle-to-short wavelength signals of the earth's gravity field in geodesy, geodynamics and marine sciences research. In recent years, some prototypes of inertial platform and strapdown marine gravimeters have been developed, where the inertial platform gravimeter systems include CHZ-II and ZL11, and strapdown gravimeter systems include SAG-2M and SGA-WZ. In order to validate the performance of these marine gravimeter prototypes, a synchronous test with the widely used gravimeters GT-2M and LCR arranged on the same vessel was carried out in the north of South China Sea. All the data are processed according to the survey standard flow, and the performance is estimated by analyzing the errors of the repeat lines and the crossover points under the same environment. The compared results show that all the six gravimeters can meet the precision requirement of marine gravity survey. Meanwhile, the precision results of the improved gravimeters can get close to the precision of gravimeter GT-2M, higher than the precision gravimeter LCR.
\end{abstract}

Keywords: Marine gravimeter, Improved gravimeters, Performance estimates, Repeat lines, Crossover points

\section{Main text Introduction}

Global ocean area accounts for $71 \%$ of the total area of the earth. The marine gravity anomaly survey plays an important role in the fields of geodesy, geodynamics and marine sciences (Ebbing et al. 2013; Sandwell et al. 2013). Marine gravimeter is the main tool for acquiring the marine gravity anomaly with the moving-based platform, which had been proved to be the primary technique to efficiently obtain middle-to-short wavelength gravity anomalies (Sandwell et al. 2013).

At present, several marine gravimeters had been widely used around the world to provide accuracies better than $2 \mathrm{mGal}$ for spatial resolutions down to $2 \mathrm{~km}$ (Liu et al. 2017; Hu et al. 2017), such as two-axis stable platform gravimeter system LCR (Lacoste and Romberg Air-Sea

\footnotetext{
*Correspondence: yuanyuan_sio@126.com

${ }^{1}$ Key Laboratory of Submarine Geosciences, Ministry of Natural

Resources, Hangzhou 310012, China

Full list of author information is available at the end of the article
}

Gravimeter), KSS-31M, MGS-6 (Marine Gravity System 6), and gimbaled inertial navigation gravimeter system, GT-2M, AIRGrav (Airborne Inertially Referenced Gravimeter), BGM3, Chekan-AM. They show up different precision levels in real applications, due to the difference in the measuring principles. A synchronized comparison test on the same platform is the most direct method to evaluate the performance of them. Studinger et al. (2008) had evaluated the relative performance and suitability of airborne gravimeters AIRGrav and GT-1A with the BGM-3 and LCR from the flight test over the Canadian Rocky Mountains near Calgary, which showed that AIRGrav and GT-1A could get higher resolution and precision than the others. Forsberg et al. (2015) compared the gravimeters LCR and Chekan-AM in Nepal, and found that two systems can obtain a good agreement result. Ouyang et al. (2013) tested 5 sea-air gravimeters with 4 different types equipped onboard the same Y-8 fixed wing aircraft by repeat lines and crossover points, which shows that airborne gravimeter GT-1A of Russia has the best technical index of comprehensive 
performance. Zhang et al. (2015), (2017) test the gravimeters GT-2M, KSS-31M and ZLS in the same vessel, and pointed out that the results of three marine gravimeters are quite different when the vessel speed and heading change or under the bad sea condition, with the gravimeter GT-2M having the best precision. By comprehensive analysis, the gravimeter GT-2M by Canadian Micro Gravity was recognized as the best to get the highest precision result in the same measuring environment.

In recent years, some prototypes of inertial platform and strapdown marine gravimeters have been developed, where the inertial platform gravimeter systems include CHZ-II and ZL11, and strapdown gravimeter systems include SAG-2M (Sea-Air Gravimeter-2 Marine) and SGA-WZ. They were made by Institute of Geodesy and Geophysics, Chinese Academy of Sciences, Tianjin Navigation Instrument Research Institute, Beijing Institute of Aerospace Control Devices, and National University of Defense Technology, respectively. All of these gravimeters have undergone many sea trials to validate their precision by crossover point and repeat lines alone (Cai et al. 2012, 2013, 2017; Luo et al. 2017; Tu et al. 2015; Wang et al. 2018; Yu et al. 2015; Zhang 2007; Zhang et al. 2019; Zhao et al. 2015; 2020). However, all these gravimeters are not compared together to validate their performance under the same sea conditions. The compared results can be useful for the users of marine gravity survey to make a choice in real applications.

In order to validate the performance of the marine gravimeters CHZ-II, ZL11, SGA-WZ and SAG-2M, this paper shows a synchronous comparison test of them with widely used commercial gravimeters GT-2M (No. 39) and LCR (No. S129) arranged on the same vessel was carried out in the north of South China Sea.

\section{System description}

In this section, the measuring principles of the marine gravimeter prototypes CHZ-II, ZL11-A, SAG-2M, SGAWZ developed from Chinese research institutes and the commercial gravimeters GT-2M and LCR are described.

The gravimeter CHZ-II in Fig. 1a is based on Hooke's law, which uses a vertical zero-length spring mounted on a two-axis stabilization platform. The gravity sensor uses an axisymmetric structure design, consisting of a vertical main spring and a tube quality, which has only one degree of freedom along its own axis of vertical movement (Tu et al. 2015).

The gravimeter ZL11 in Fig. 1b consists of a two-axis Schuler-tuned inertial stabilized platform with three fiber-optic gyroscopes and three acceleration sensors. A gravity sensor replaces the vertical accelerometer. The gravity sensor is an axial system with a reference
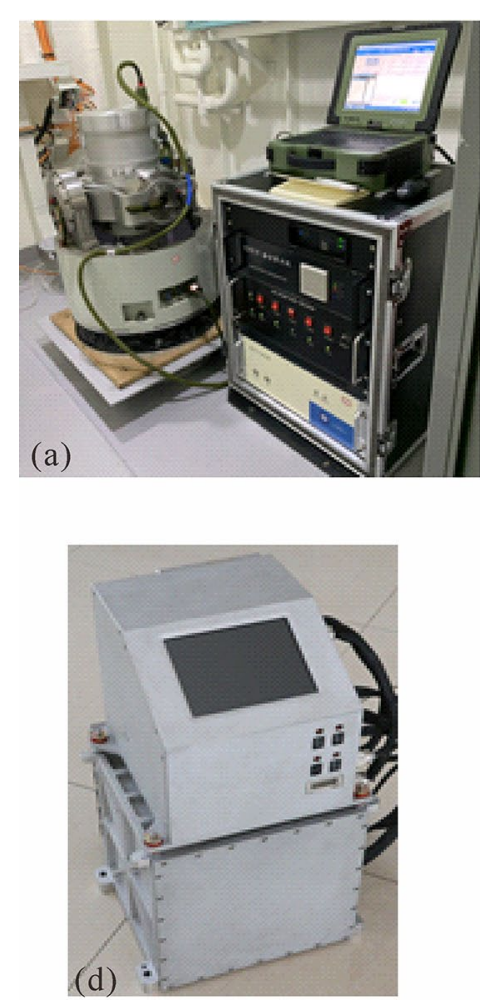
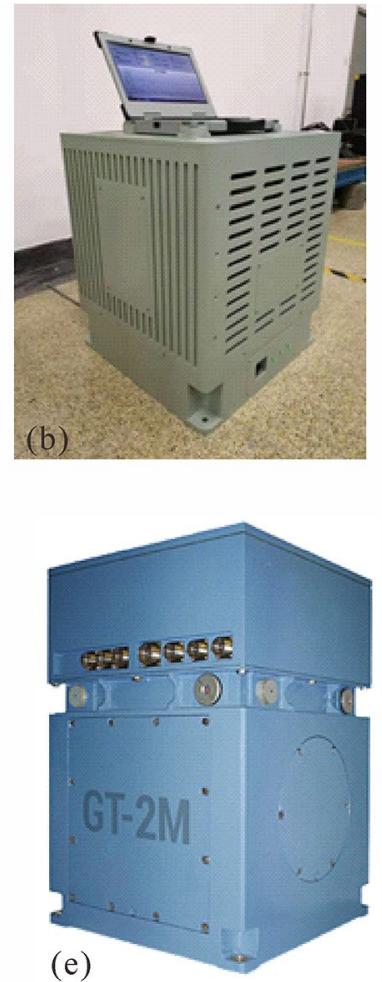

(e)

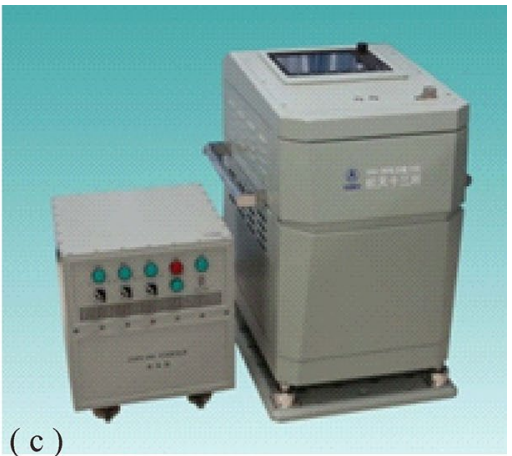

(f)

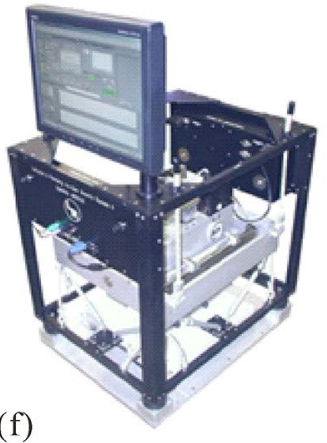

Fig. 1 Marine gravimeters: a CHZ-II; b ZL11; c SAG-2M; d SGA-WZ; e GT-2M; f LCR 
mass on a flexible support and an electromagnetic feedback. This design minimizes the cross-coupling effect caused by horizontal acceleration and obtains the gravity anomaly in real time (Zhao et al. 2020; Zhang et al. 2019).

The strapdown gravimeters SAG-2M in Fig. 1c and SGA-WZ in Fig. 1d are both based on Newton's second law, which obtains gravity acceleration and carrier attitude information through triaxial accelerometers and gyroscopes, and then realizes gravity anomaly extraction from a combination of accelerometers and a kinematic navigation system DGPS in inertial reference frame (Cai et al. 2012, 2013, 2017; Luo et al. 2017; Wang et al. 2018; Yu et al. 2015).

The gravimeter GT-2M in Fig. 1e is a new marine gravimeter, which has evolved from the highly successful GT-1A and GT-2A airborne gravimeters. It has a vertically oriented sensor, vertical scalar GPS-INS gravimeter with a Schuler-tuned three-axis inertial platform. A turntable provides the platform's azimuth axis control (Gabell et al. 2004; Olson et al. 2011).

The gravimeter LCR in Fig. If consists of a highly damped, spring-type gravity sensor mounted on a gyro-stabilized platform with associated electronics for obtaining gravity readings. However, the cable-stayed zero-length spring will lead to the cross-coupling effect, which cannot be removed under bad sea conditions (LaCoste et al. 1967; 1982).

\section{Measurement process}

Between 4 June 2018 and 25 June 2018, a synchronous comparing test was carried out in the north of South China Sea, with gravimeters CHZ-II, SAG-2M, SGA-WZ, ZL11, GT-2M (No. 39) and LCR (No. S129) arranged on the same vessel, Xiangyanghong No. 06 Comprehensive Scientific Research Vessel, Fig. 2.

The gravimeters were all installed along the central axis of the vessel in the middle part of it, which is the most stable area on the vessel. The maximum deviation distance from the central axis should not exceed $5 \mathrm{~m}$. During the survey, the navigation position data for all the gravimeters came from the same GNSS (Global Navigation Satellite System) receiver SF3050, installed on the top of the vessel to avoid signal blocking. A vessel coordinate system was constructed to correct the lever-arm effects of different gravimeters to make the position of the GNSS coincide exactly with the position of the gravimeters (Jekeli 2000; Li 2013). Table 1 shows the coordinates of the gravimeters and GNSS in the vessel coordinate system.

The survey lasted 22 days at an average sailing speed of 10 knots. We completed 13 effective gravity profiles, shown in Fig. 3, with line L1, L2 and L3 having been round-trip measured three times, line L6 having been round-trip measured two times, and getting 39 crossover points. The north to south repeat lines design can reduce the Eötvös effect from the earth rotation, which

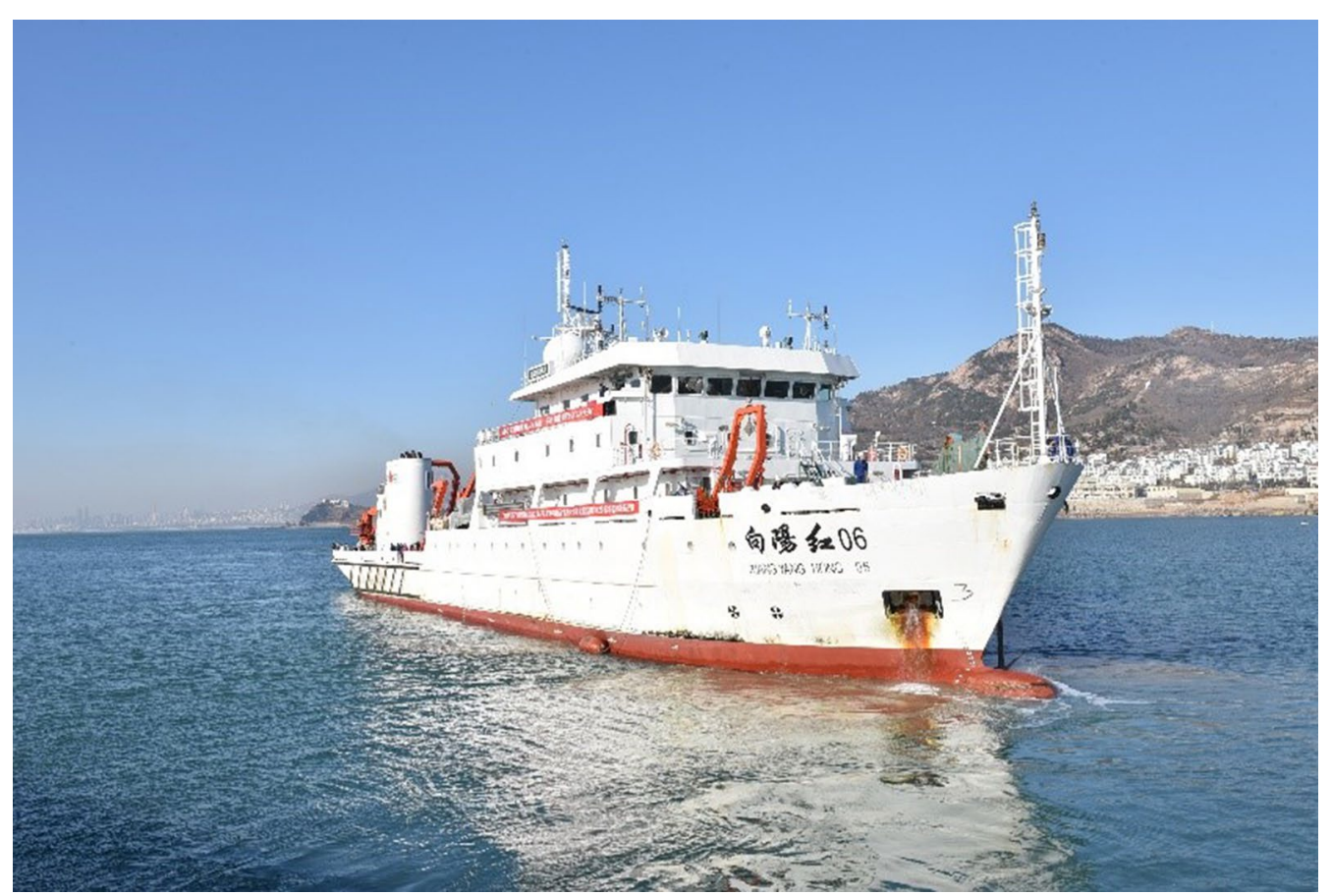

Fig. 2 Survey carrier: Xiangyanghong No. 06 Vessel 
Table 1 Position coordinates of sensors in the vessel coordinate system

\begin{tabular}{lllcc}
\hline No. & Sensors & $\begin{array}{l}\boldsymbol{X} \text { coordinate } \\
(\mathbf{m})\end{array}$ & $\begin{array}{l}\boldsymbol{Y} \text { coordinate } \\
(\mathbf{m})\end{array}$ & $\boldsymbol{Z}$ coordinate $(\mathbf{m})$ \\
\hline 1 & CHZ-II & 46.990 & 2.197 & 0.429 \\
2 & SAG-2M & 45.082 & 1.318 & 0.249 \\
3 & SGA-WZ & 49.246 & 1.226 & 0.399 \\
4 & ZL11 & 48.695 & 1.333 & 0.588 \\
5 & GT-2M & 50.074 & 1.351 & 0.778 \\
6 & LCR & 48.031 & -0.278 & -2.411 \\
7 & GNSS & 56.052 & 2.174 & 13.007 \\
\hline
\end{tabular}

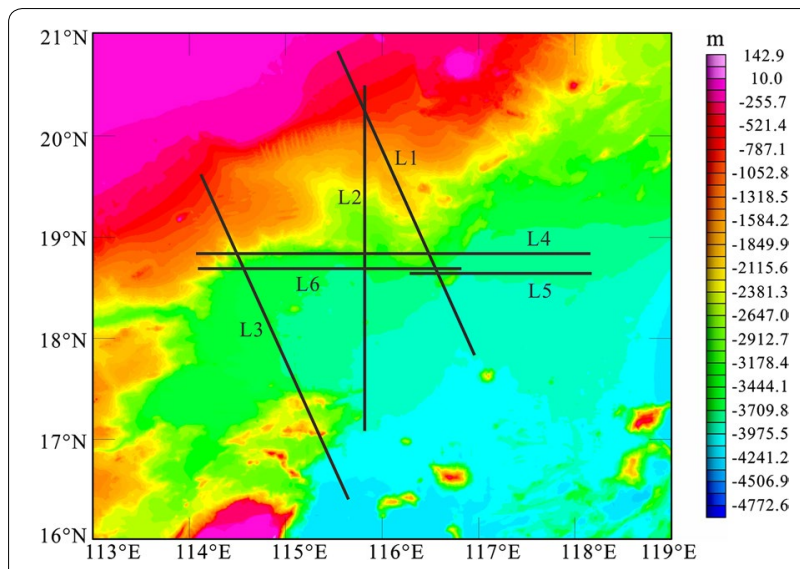

Fig. 3 Bathymetry of the area and distribution of effective measured lines

can better reflect the performance characteristics of the gravimeter itself. However, gravimeter CHZ-II could get only 11 effective lines without L2-3 and L4, because of a failure of the temperature control system.

\section{Data processing}

In order to compare the performance of the gravimeters fairly, we established a unified process standard. All gravity raw data were processed by zero drift correction, Eötvös correction and normal gravity correction to obtain the free-air gravity anomaly in CGCS 2000 (China Geodetic Coordinate System 2000).

Since the measuring principle of each gravimeter is different, each gravimeter has its own processing mode. The gravimeters $\mathrm{CHZ}-\mathrm{II}$ and $\mathrm{ZL}-11$ have the same processing flow with LCR, because their measured raw data are only filtered by the low-pass filter without any corrections.

For gravimeter GT-2M, the measured raw data is a free-air gravity anomaly, which has been filtered by three different filter length, $150 \mathrm{~s}, 300 \mathrm{~s}$ and $600 \mathrm{~s}$, and has the Eötvös effect and normal gravity effect to be corrected. However, the normal gravity correction formula of GT-2M,

$$
\begin{aligned}
g_{i}= & 978030\left(1+0.005302 \sin ^{2} \varphi_{i}\right. \\
& \left.-0.000007 \sin ^{2}\left(2 \varphi_{i}\right)\right)-14
\end{aligned}
$$

is the Helmert equation in the Potsdam reference ellipsoid, which is different from the normal gravity correction formula in CGCS 2000:

$$
\begin{aligned}
g_{i}= & 978032.53349\left(1+0.00530244 \sin ^{2} \varphi_{i}\right. \\
& \left.-0.00000582 \sin ^{2} 2 \varphi_{i}\right)
\end{aligned}
$$

Therefore, in order to obtain the free-air gravity anomaly in CGCS 2000, a transformation is needed (Zhang et al. 2017).

Unlike the other gravimeters, the strapdown gravimeters SAG-2M and SGA-WZ directly measure the specific force and the angular motion information of the carrier system. Based on the Newton's second law, gravity can be extracted from a combination of the measured specific force information and the kinematic acceleration of the carrier measured by GNSS. The gravity anomaly calculation formula of the strapdown gravimeter is

$$
\begin{aligned}
\delta g_{D}= & \dot{v}_{D}-f_{D}+\left(2 w_{i e} \cos \varphi+\frac{v_{E}}{R_{N}+h}\right) \\
& \times v_{E}+\frac{v_{N}^{2}}{R_{M}+h}-r
\end{aligned}
$$

where $\dot{v}_{D}$ is the down component of acceleration of the carrier, $f_{D}$ is the down component of the specific force, $w_{i e}$ is the angular velocity of the Earth's rotation, $\varphi$ is the latitude of the carrier's location, $R_{M}$ and $R_{N}$ denotes the meridian radius and the prime vertical radius at the point on the reference ellipsoid obtained by orthogonal projection of the measuring point in the direction of the ellipsoid normal, respectively, $h$ is the height, and $v_{E}$ and $v_{N}$ are the eastbound and northbound speed of the carrier, respectively.

Zhao et al. (2015) and Wang et al. (2018) has pointed that the precision of the gravity anomaly extraction depends on the precision of the specific force, which mainly relies on the measurement precisions of the attitude matrix of the carrier and the specific force in the carrier coordinate system during navigation. Due to the random error of the gyroscope and the accelerometer in the strapdown gravimeter drifts over time, a combined navigation approach with a Kalman filter is adopted to correct for the attitude error caused by the drift of the inertial instrument. A detailed strapdown 

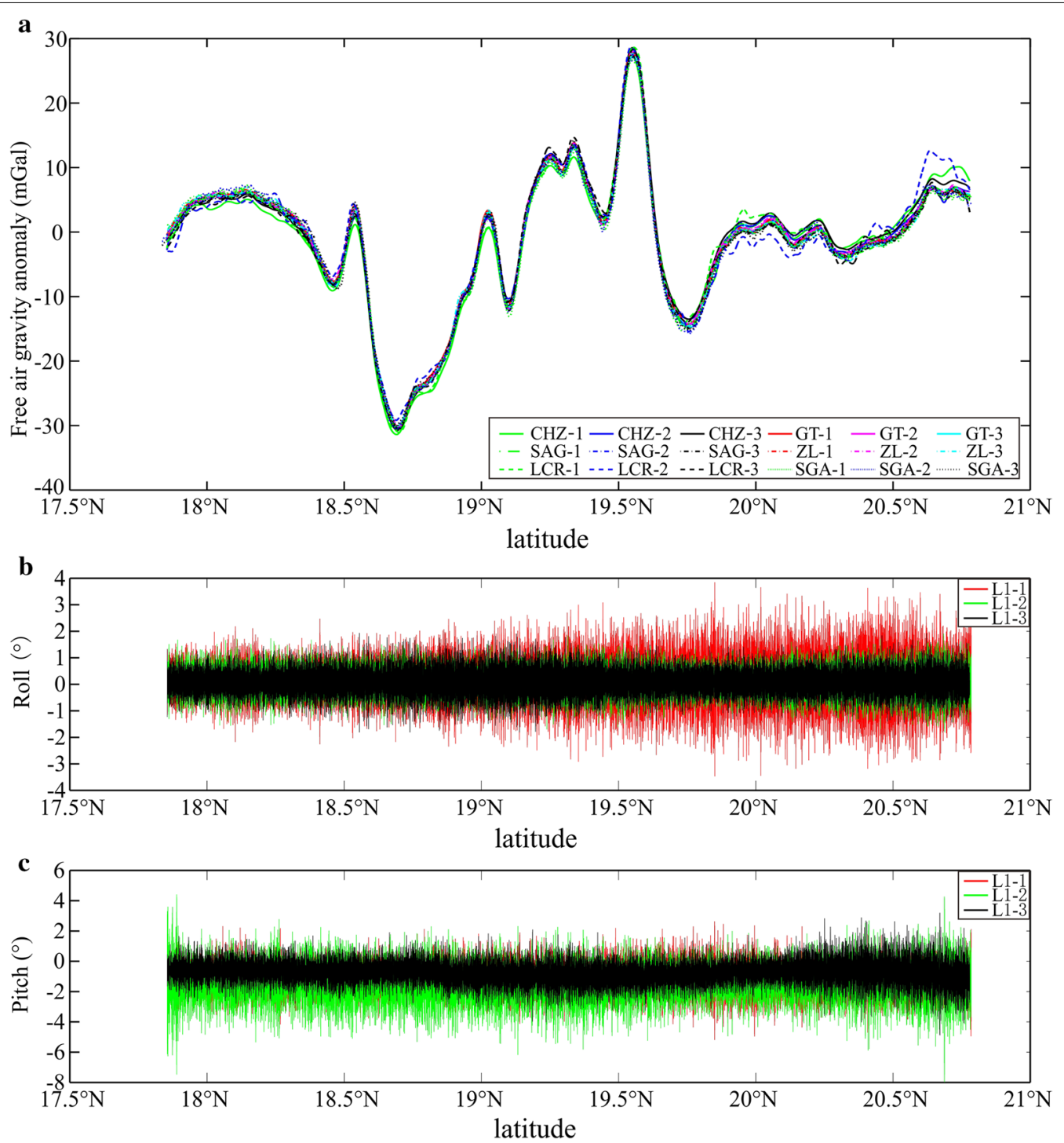

Fig. 4 Comparison of free-air gravity anomaly (a) results from measurements collected with all the gravimeters on the three times repeated Line 1 (ref. to Fig. 3); b, c sea conditions during the survey can be envisaged from roll and pitch vessel data

gravimeter data processing flow is demonstrated by Zhao et al. (2015) and Wang et al. (2018).

\section{Survey data comparison}

In order to evaluate the performance of the gravimeters, the root mean square error (RMS), systematic deviation (SD) and mean error (ME) of the repeat lines and the crossover points are the main technology specifications to determine their precision and internal consistency (Guo et al. 2008; Huang et al. 2013; Yu et al. 2015). The root mean square error describes the precision of the observed data. The systematic deviation is the arithmetic mean of the observation error, which reflects the systemic change characteristics of observation error. The mean error is the average of the absolute value of the observation error, which describes the discrete degree of the observation error.

For the multi-measured repeat line, we use a combination of any two of the repeat times to estimate the RMS precision, which can reflect the reliability and stability of the gravimeter more comprehensively. The RMS precision of any two repeat lines can be calculated by Eqs. 4, 5: 

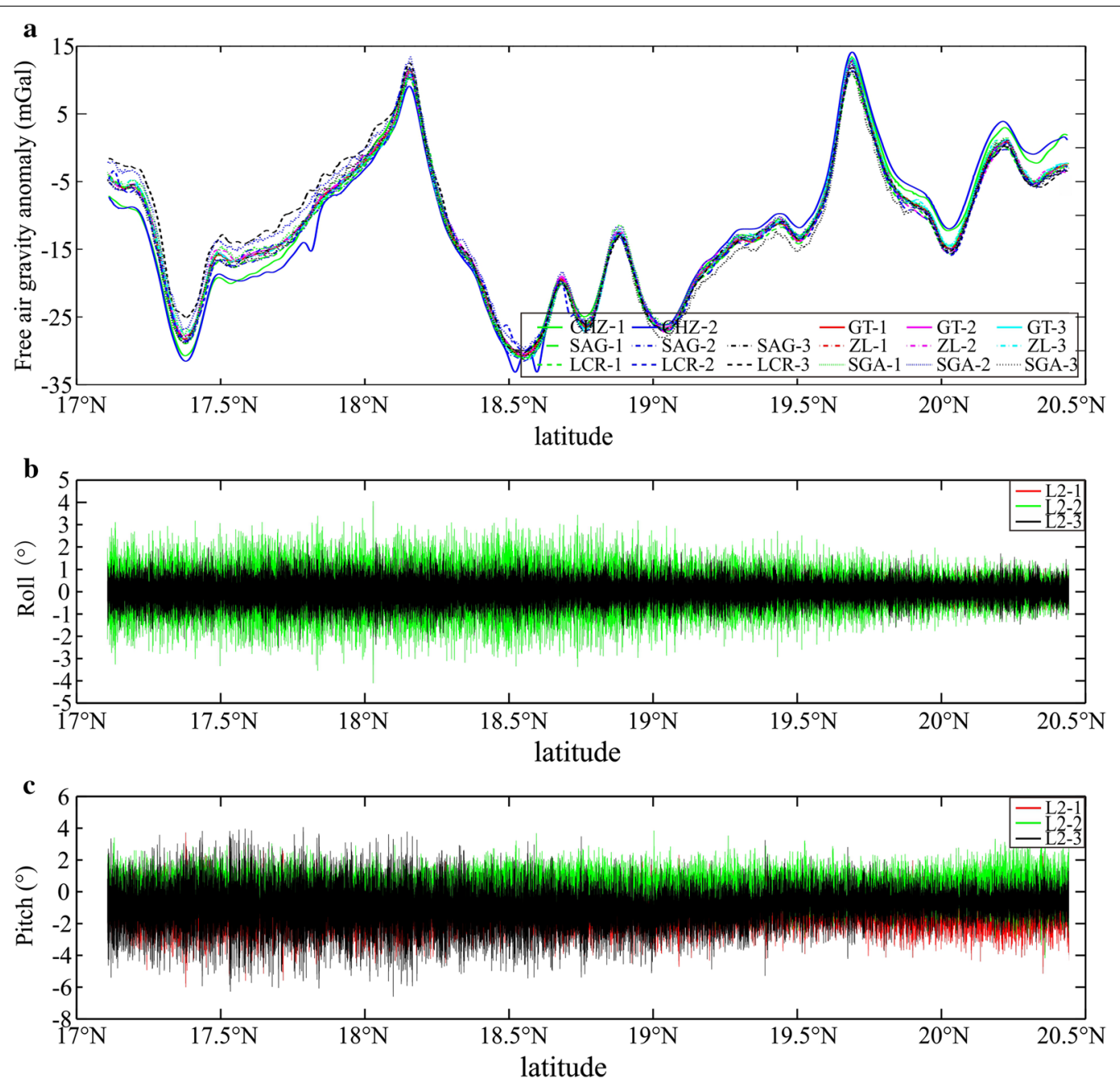

Fig. 5 Comparison of free-air gravity anomaly (a) results from measurements collected with all the gravimeters on the three times repeated Line 2 (ref. to Fig. 3); b, c sea conditions during the survey can be envisaged from roll and pitch vessel data

$$
\begin{gathered}
\sigma_{R M S}= \pm \sqrt{\frac{\sum_{i=1}^{n}\left(d_{i 12}\right)^{2}}{2 n}}, \\
d_{i 12}=g_{i 1}-g_{i 2},(i=1,2,3, \ldots, n),
\end{gathered}
$$

where $d_{i 12}$ is the difference between two measurement value $g_{i 1}$ and $g_{i 2}$ in the repeat line $i, n$ is the number of observed points in each repeat line.

The SD of repeat line can be calculated by Eq. 6:

$$
\sigma_{S D}=\frac{\sum_{i=1}^{n} d_{i 12}}{2 n} .
$$

The ME of repeat line can be calculated by Eq. 7:

$$
\sigma_{M E}=\frac{\sum_{i=1}^{n}\left|d_{i 12}\right|}{\sqrt{2} n} .
$$

For the crossover points, the RMS error can be calculated by Eq. 8 :

$$
\sigma_{R M S}= \pm \sqrt{\frac{\sum_{i=1}^{m} d_{i}^{2}}{2 m}}(i=1,2, \ldots, m),
$$

where $d$ is the discrepancy of intersection point gravity anomaly, and $m$ is the number of intersection points.

The SD error can be calculated by Eq. 9:

$$
\sigma_{S D}=\frac{1}{2 m} \sum_{i=1}^{m} d_{i}
$$



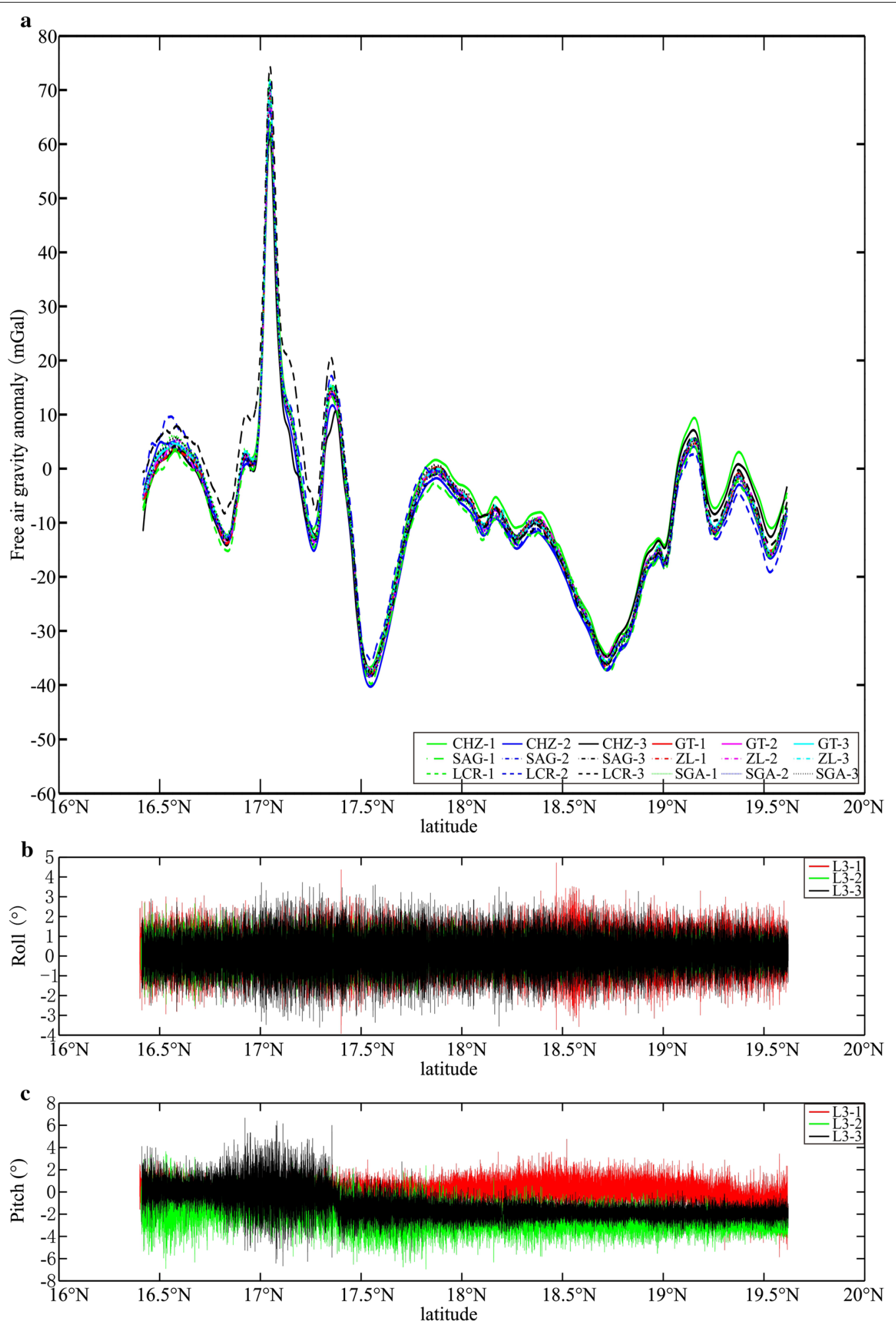

Fig. 6 Comparison of free-air gravity anomaly (a) results from measurements collected with all the gravimeters on the three times repeated Line 3 (ref. to Fig. 3); b, c sea conditions during the survey can be envisaged from roll and pitch vessel data 
Table 2 Error statistics of different gravimeters on the repeated line L1

\begin{tabular}{|c|c|c|c|c|c|c|c|c|c|c|c|c|c|c|c|c|c|c|}
\hline \multirow[t]{2}{*}{ Times } & \multicolumn{3}{|c|}{ GT-2M 39} & \multicolumn{3}{|l|}{ CHZ-II } & \multicolumn{3}{|c|}{ LCR S129 } & \multicolumn{3}{|c|}{ SAG-2M } & \multicolumn{3}{|c|}{ SGA-WZ } & \multicolumn{3}{|l|}{ ZL11 } \\
\hline & $1-2$ & $1-3$ & $2-3$ & $1-2$ & $1-3$ & $2-3$ & $1-2$ & $1-3$ & $2-3$ & $1-2$ & $1-3$ & $2-3$ & $1-2$ & $1-3$ & $2-3$ & $1-2$ & $1-3$ & $2-3$ \\
\hline RMS (mGal) & 0.18 & 0.17 & 0.17 & 0.91 & 0.79 & 0.48 & 1.51 & 0.77 & 1.25 & 0.14 & 0.17 & 0.16 & 0.57 & 0.63 & 0.68 & 0.29 & 0.35 & 0.49 \\
\hline $\mathrm{SD}$ (mGal) & 0.06 & 0.09 & 0.04 & -0.24 & -0.37 & -0.13 & -0.08 & 0.04 & 0.12 & 0.01 & 0.07 & 0.06 & -0.10 & -0.13 & -0.03 & -0.04 & 0.11 & 0.14 \\
\hline ME (mGal) & 0.16 & 0.14 & 0.14 & 0.78 & 0.69 & 0.33 & 1.23 & 0.64 & 0.95 & 0.12 & 0.14 & 0.12 & 0.45 & 0.54 & 0.57 & 0.24 & 0.28 & 0.42 \\
\hline
\end{tabular}

Table 3 Error statistics of different gravimeters on the repeated line L2

\begin{tabular}{|c|c|c|c|c|c|c|c|c|c|c|c|c|c|c|c|c|c|c|}
\hline \multirow[t]{2}{*}{ Times } & \multicolumn{3}{|c|}{ GT-2M 39} & \multicolumn{3}{|c|}{ CHZ-II } & \multicolumn{3}{|c|}{ LCR S129 } & \multicolumn{3}{|c|}{ SAG-2M } & \multicolumn{3}{|c|}{ SGA-WZ } & \multicolumn{3}{|l|}{ ZL11 } \\
\hline & $1-2$ & $1-3$ & $2-3$ & $1-2$ & $1-3$ & $2-3$ & $1-2$ & $1-3$ & $2-3$ & $1-2$ & $1-3$ & $2-3$ & $1-2$ & $1-3$ & $2-3$ & $1-2$ & $1-3$ & $2-3$ \\
\hline RMS (mGal) & 0.16 & 0.23 & 0.30 & 0.89 & - & - & 0.59 & 1.41 & 1.24 & 0.31 & 0.23 & 0.42 & 0.79 & 0.97 & 1.26 & 0.36 & 0.33 & 0.51 \\
\hline $\mathrm{SD}$ (mGal) & -0.06 & 0.02 & 0.08 & 0.11 & - & - & -0.17 & -0.65 & -0.47 & 0.18 & -0.01 & -0.18 & -0.22 & 0.48 & 0.70 & -0.02 & -0.03 & -0.02 \\
\hline ME (mGal) & 0.14 & 0.19 & 0.25 & 0.72 & - & - & 0.45 & 1.02 & 0.86 & 0.26 & 0.18 & 0.35 & 0.63 & 0.81 & 1.12 & 0.28 & 0.26 & 0.41 \\
\hline
\end{tabular}

Table 4 Error statistics of different gravimeters on the repeated line L3

\begin{tabular}{|c|c|c|c|c|c|c|c|c|c|c|c|c|c|c|c|c|c|c|}
\hline \multirow[t]{2}{*}{ Times } & \multicolumn{3}{|c|}{ GT-2M 39} & \multicolumn{3}{|c|}{ CHZ-II } & \multicolumn{3}{|c|}{ LCR S129 } & \multicolumn{3}{|c|}{ SAG-2M } & \multicolumn{3}{|c|}{ SGA-WZ } & \multicolumn{3}{|l|}{ ZL11 } \\
\hline & $1-2$ & $1-3$ & $2-3$ & $1-2$ & $1-3$ & $2-3$ & $1-2$ & $1-3$ & $2-3$ & $1-2$ & $1-3$ & $2-3$ & $1-2$ & $1-3$ & $2-3$ & $1-2$ & $1-3$ & $2-3$ \\
\hline RMS (mGal) & 0.36 & 0.18 & 0.30 & 2.52 & 1.47 & 1.86 & 2.05 & 3.08 & 2.40 & 0.42 & 0.36 & 0.29 & 0.42 & 0.86 & 0.85 & 0.60 & 0.52 & 0.60 \\
\hline SD (mGal) & 0.18 & 0.04 & -0.13 & 1.50 & 0.75 & -0.76 & -0.71 & -1.56 & -0.86 & 0.08 & 0.01 & -0.07 & -0.01 & -0.31 & -0.32 & -0.07 & -0.27 & -0.20 \\
\hline ME (mGal) & 0.29 & 0.15 & 0.24 & 2.35 & 1.11 & 1.65 & 1.65 & 2.32 & 1.84 & 0.35 & 0.29 & 0.20 & 0.34 & 0.67 & 0.62 & 0.49 & 0.42 & 0.47 \\
\hline
\end{tabular}

The ME error can be calculated by Eq. 10:

$$
\sigma_{M E}=\frac{1}{\sqrt{2} m} \sum_{i=1}^{m}\left|d_{i}\right| .
$$

Figures 4, 5, 6 compare the measured results of the repeat lines L1, L2 and L3 in different sea conditions, all with three round-trip measurements. We can see that the measured gravity anomalies of the improved gravimeters CHZ-II, SAG-2M, SGA-WZ and ZL11 had a good coincident with the measured gravity anomalies of gravimeter GT-2M in every repeat line, with slightly different. The gravimeter LCR had the biggest anomalies from the others, which means that the gravimeter LCR had a worst result in the comparison. The main reason for this is that the gravimeter LCR has a strong crosscoupling effect affected by the horizontal accelerations in the rough sea condition (Ouyang et al. 2011; Wang et al. 2018; Zhang et al. 2007), with large roll and pitch angles, especially when the pitch angle is rough, shown in Figs. 4 and 6. Genrich and Minster (1991) pointed that the noise caused by the heave and roll of the vessel due to wave action can be well removed by the low-pass filter of the gravimeter. Therefore, pitch angle is the main reason leading to the poor results of gravimeter LCR. In repeat line L2, the gravimeter CHZ-II only had two effective measurements in the lines L2-1 and L2-2, without the $\mathrm{L} 2-3$, as the failure originates from the temperature control system. This also led to three false anomalies in the line L2-2 and systematic deviation compared to the others in the lines L2-1 and L2-2 in Fig. 5.

Tables 2, 3, 4 describe the RMS, SD and ME errors statistics results of a combination of two of repeat times of different gravimeters in the repeat lines L1, L2 and L3, indicating that all the six gravimeters can obtain the precision requirement within $2 \mathrm{mGal}$ of marine gravity measurement. However, gravimeter GT-2M had the minimum RMS, SD and ME statistic results of all the gravimeters in the repeat lines L1, L2 and L3, with the maximum RMS being $0.36 \mathrm{mGal}$, SD $0.18 \mathrm{mGal}$ and ME $0.29 \mathrm{mGal}$. The improved gravimeters CHZ-II, SAG-2M, SGAWZ and ZL11 all had the RMS, SD and ME statistics results less than the gravimeter LCR in the repeat lines. Therefore, we can conclude that the gravimeter GT-2M showed the best performance in different sea conditions; improved gravimeters CHZ-II, SAG-2M, SGA-WZ and ZL11 were better than gravimeter LCR.

Additionally, we also used the crossover points statistic errors to estimate the performance of different gravimeters. Figure 7 shows the crossover points error 

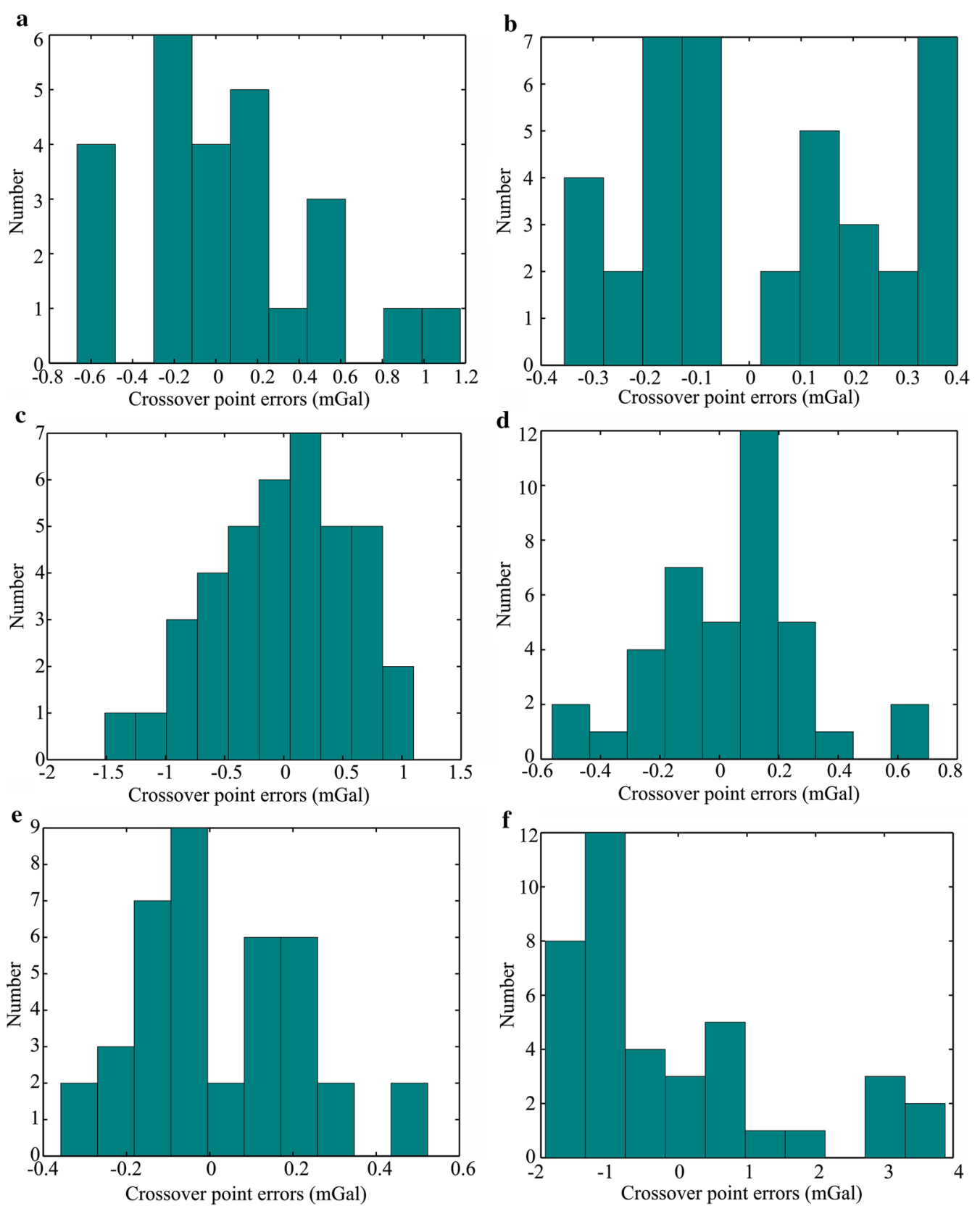

Fig. 7 Crossover point errors distribution for the different gravimeters: a CHZ-II; b SAG-2M; c SGA-WZ; d ZL11; e GT-2M; $\mathbf{f} L C R$

distributions for the different gravimeters. Table 5 indicates the maximum error, minimum error, RMS, SE and ME statistic results. We can see that the gravimeter LCR had the largest crossover errors than the others, with the maximum error being $3.88 \mathrm{mGal}$, and the minimum error being $-1.94 \mathrm{mGal}$. The crossover point errors of the other gravimeters were between $-1.5 \mathrm{mGal}$ and 1.2 mGal. From the RMS statistics results, we can see that the gravimeters GT-2M, CHZ-II, SAG-2M, SGA-WZ and ZL11 all can have a RMS precision better than 0.5 $\mathrm{mGal}$ and the gravimeter LCR a RMS precision with 1.11 $m G$ al. From the SD statistics results, we can see that all the gravimeters showed little system observation errors. From the ME statistics results, the discrete degree of the observation error of gravimeters CHZ-II, SAG-2M, SGAWZ, ZL11 and GT-2M are more stable than the gravimeter LCR. 
Table 5 Crossover points error statistics for the different gravimeters

\begin{tabular}{|c|c|c|c|c|c|c|}
\hline & CHZ-II & SAG-2M & SGA-WZ & ZL11 & GT-2M 39 & LCR S129 \\
\hline Effective lines & 11 & 13 & 13 & 13 & 13 & 13 \\
\hline Crossover points number & 25 & 39 & 39 & 39 & 39 & 39 \\
\hline Maximum value (mGal) & 1.18 & 0.40 & 1.10 & 0.70 & 0.52 & 3.88 \\
\hline Minimum value (mGal) & -0.67 & -0.36 & -1.51 & -0.56 & -0.36 & -1.94 \\
\hline RMS (mGal) & 0.32 & 0.17 & 0.41 & 0.18 & 0.14 & 1.11 \\
\hline $\mathrm{SD}$ (mGal) & 0.02 & 0.01 & 0.01 & 0.02 & 0.01 & -0.06 \\
\hline ME (mGal) & 0.24 & 0.15 & 0.33 & 0.14 & 0.11 & 0.91 \\
\hline
\end{tabular}

\section{Conclusions}

In this paper, we have compared the performance of the improved marine gravimeters CHZ-II, SAG-2M, SGAWZ and ZL11 developed in Chinese research institutions with the widely used marine gravimeters GT-2M by Canadian Micro Gravity and LCR S129 by LaCoste \& Romberg in side-by-side testing. The purpose of the test program was to evaluate the suitability of these improved systems for research applications by designing different profiles, i.e., measurement surveys conducted on repeated lines and crossover points under different sea conditions. The results show that the gravimeter GT-2M can get the best performance in the rough sea condition with the minimum RMS, SD and $\mathrm{ME}$ in the statistic errors of the repeat lines and crossover points. This test has demonstrated that the homemade marine gravimeter prototypes CHZ-II, SAG-2M, SGA-WZ and ZL11 can get similar precision results to the gravimeter GT-2M with RMS precision less than $0.5 \mathrm{mGal}$, better than the gravimeter LCR. The main findings of this study clearly indicate that the gravimeters we have tested can compete with the accuracy standards currently achieved by the best instruments available on the world market for marine gravimetry.

\section{Abbreviations}

RMS: Root mean square error; SD: Systematic deviation; ME: Mean error; GNSS: Global Navigation Satellite System.

\section{Acknowledgements}

We express our sincere gratitude to the editors and reviewers in improving the manuscript, and acknowledge the Institute of Geodesy and Geophysics, Chinese Academy of Sciences, Beijing Institute of Aerospace Control Devices, National University of Defense Technology, and Tianjin Navigation Instrument Research Institute in providing the improved gravimeters to the comparison test

\section{Authors' contributions}

In this study, YY and JG conceived and designed the experiments. YY, ZW and $Z S$ were responsible for the initial maritime data collection. YY processed the data and wrote the main manuscript text. All authors read and approved the final manuscript.

\section{Funding}

This study is supported by the Fundamental Research Funds for National Natural Science Foundation of China (41804169), Natural Science Foundation of Zhejiang Province, China (LQ18D040001), Basic Scientific Research Special Fund Project of Second Institute of Oceanography, MNR (JP1902), National key research and development plan (SQ2018YFF020145), Marine/Airborne Gravimeter Research Project (2011YQ12004505), Open Fund of MLR Key Laboratory of Marine Mineral Resources, MNR,(KLMMR-2017-B-03).

\section{Availability of data and materials}

The datasets used and/or analyzed during the current study are available from the corresponding author on any request.

\section{Competing interests}

The authors declare that they have no competing interests.

\section{Author details}

${ }^{1}$ Key Laboratory of Submarine Geosciences, Ministry of Natural Resources, Hangzhou 310012, China. ${ }^{2}$ Second Institute of Oceanography, Ministry of Natural Resources, Hangzhou 310012, China.

Received: 5 May 2020 Accepted: 23 June 2020

Published online: 02 July 2020

\section{References}

Cai S, Zhang K, Wu M, Huang Y (2012) Long-term stability of the SGA-WZ strapdown airborne gravimeter. Sensors 12:11091-11099

Cai SK, Wu MP, Zhang KD, Cao JL, Tuo ZH, Huang YM (2013) The first airborne scalar gravimetry system based on SINS/DGPS in China. Sci China Earth Sci. 56:2198-2208

Cai S, Tie J, Zhang K, Cao J, Wu M (2017) Marine gravimetry using the strapdown gravimeter SGA-WZ. Mar Geophys Res 38:325-340

Ebbing J, Bouman J, Fuchs M, Lieb V, Haagmans R, Meekes J, Fattah RA (2013) Advancements in satellite gravity gradient data for crustal studies. Lead Edge 8:900-906

Forsberg R, Olesen AV, Einarsson I (2015) Airborne gravimetry for geoid determination with Lacoste Romberg and Chekan gravimeters. Gyroscopy Navigation 6:265-270

Gabell A, Tuckett H, Olson D (2004) The GT-1A mobile gravimeter. Australian SEG Airborne Gravity Workshop, pp. 55-62

Genrich JF, Minster JB (1991) Near-real time reduction of vessel board gravity using Kalman-filtered GPS measurements. Geophysics 56(12):1971-1979

Guo ZH, Xiong SQ, Zhou JX, Zhou XH (2008) The research on quality evaluation method of test repeat lines in airborne gravity survey. Chin J Geophysics 51:1538-1543

Hu PH, Zhao M, Huang H, Liu DB, Tang JH, Wei C (2017) Review on the development of airborne/marine gravimetry instruments. Navigation Positioning Timing 4:10-19

Huang MT, Ouyang YZ, Zhai GJ, Lu P (2013) Comment on the formulas of accuracy evaluation for multi-line overlapping measurements in 
ship-borne and air-borne gravity survey. Geomatics Inf Sci Wuhan Univ 38(10):1175-1177

Jekeli C (2000) Inertial navigation systems with geodetic applications. W. deGruyter, Berlin

LaCoste L, Clarkson N, Hamilton G (1967) LaCoste \& romberg stabilized platform vessel board gravity meter. Geophysics 32:99-109

LaCoste L, Ford J, Bowles R, Archer K (1982) Gravity measurements in an airplane using state-of-the-art navigation and altimetry. Geophysics 47:832-838

Li X (2013) Examination of two major approximations used in the scalar airborne gravimetric system: a case study based on the LCR system. J Geod Sci. 3:32-39

Liu M, Huang MT, Ouyang YZ, Deng KL, Zhai GJ, Lu XP, Wu TQ, Chen X (2017) Development and prospect of air-sea gravity survey and its applications, part II: sensor, plan and design of survey. Hydrographic Surveying Charting 37:1-11

Luo C, Xue ZB, Li DM, Li HB, Wang WJ, Ma CZ (2017) Applications and data processing of strapdown gravimeter in marine gravity survey. Navigation Positioning Timing 4:36-42

Olson D, Jurist S, Smoller J (2011) GT-2M technology technical note. Canadian Micro Gravity Ltd, Toronto

Ouyang YZ, Lu XP, Huang MT, Zhai GJ (2011) An integrated method for compensating the systematic errors of marine and airborne measurements from L\&R gravimeter. Geomatics Inf Sci Wuhan Univ 36(5):625-629

Ouyang YZ, Deng KL, Lu XP, Wu TQ, Yan XC, Chang GB (2013) Tests of multitype airborne gravimeters and data analysis. Hydrographic Surveying Charting 33:6-11

Sandwell D, Garcia E, Soofi K, Wessel P, Chandler M, Smith W (2013) Toward 1-mGal accuracy in global marine gravity from CryoSat-2, Envisat, and Jason-1. Lead Edge 8:892-899

Studinger M, Bell R, Frearson N (2008) Comparison of AIRGrav and GT-1A airborne gravimeters for research applications. Geophysics 73:151-161

Tu HB, He JG, Liu LJ, Dong QQ, Hu M, Liu LT (2015) Performance characteristics for the g-sensitive elastic structure of $\mathrm{CHZ}-\mathrm{Il}$ sea gravimeter. J Geodesy Geodynamics 35(4):711-714
Wang W, Gao J, Li D, Zhang T, Luo X, Wang J (2018) Measurements and accuracy evaluation of a strapdown marine gravimeter based on inertial navigation. Sensors 18(11):3902

Yu RH, Cai SK, Wu MP, Cao JL, Zhang KD (2015) An SINS/GNSS ground vehicle gravimetry test based on SGA-WZ02. Sensors 15:23477-23495

Zhang KD (2007) Research on the methods of airborne gravimetry based on SINS/DGPS. Ph.D. Thesis, National University of Defense Technology, Changsha, China

Zhang T, Gao J, Chen M (2007) Analyzing and correcting S model gravimeter's data with correlation analysis method. Hydrographic Surveying Charting 27(2):1-5

Zhang XY, Xu X, Liao KX, Wang JS, Guan YX (2015) Results analysis for different types of gravimeters in sea trials. Hydrographic Surveying Charting 35(5):71-78

Zhang ZY, Guan YX, Xu X, Liao KX, Wang JS (2017) Some questions about data processing of GT-2 $\backslash \mathrm{M}$ marine gravimeter. Hydrographic Surveying Charting 37(1):30-33

Zhang XY, Gao W, Guan YX (2019) Discussion for method of Eötvös correction of ZL11 A gravimeter. Hydrographic Surveying Charting 39(6):21-24

Zhao L, Forsberg R, Wu M, Olesen A, Zhang K, Cao J (2015) A flight test of the strapdown airborne gravimeter SGA-WZ in greenland. Sensors 15:13258-13269

Zhao XM, Gao W, Li D, Zhang ZS (2020) Realization and application of inertial navigation platform gravity measurement technology. Navigation Positioning Timing 7(2):11-17

\section{Publisher's Note}

Springer Nature remains neutral with regard to jurisdictional claims in published maps and institutional affiliations.

\section{Submit your manuscript to a SpringerOpen ${ }^{\circ}$ journal and benefit from:}

- Convenient online submission

- Rigorous peer review

- Open access: articles freely available online

- High visibility within the field

- Retaining the copyright to your article

Submit your next manuscript at $\boldsymbol{\nabla}$ springeropen.com 